\title{
Investigation of possibility of hopper cars unloading on the car dumper VRS-134M
}

\author{
Stepan Dovhaniuk ${ }^{1}$, Volodymyr Kalashnyk $^{1}$, Alexei Reidemeister ${ }^{1, *}$, and Oleksandr Shykunov ${ }^{1}$ \\ ${ }^{1}$ Dnipro National University of Railway Transport named after Academician V. Lazaryan, 49000 Dnipro, Ukraine
}

\begin{abstract}
It was developed a computational model to evaluate the body strength of short base hopper models 19-758-01, 20-471, and 20-4015 when unloading them on the car dumper VRS-134M. Modeling of the stress-strain state of the car design was made using the finite element method. It was established that in terms of strength, bodies of the hopper models 19-758-01, 20-471, and 20-4015 are suitable for unloading on car dumper. The results of experimental studies showed sufficiently high accuracy of the chosen computational model for estimating the stress-strain state of design and confirmed the possibility of hopper car unloading on the car dumper VRS-134M.
\end{abstract}

\section{Introduction}

Open-type hopper cars of the models 19-758-01, 20-471, $20-4015$ [1-2] have a typical design for this class of rolling stock. Their bodies consist of a frame (center sill, body bolster and end beams), lateral, inclined end walls and unloading bins closed by hatches from the outside. To the side wall frame formed by the top and bottom chords, posts and trusses, plating is attached. The fastening can be rigid, like that in a car for cement transportation, model 19-758-01, or movable, like that in a car for pellet transportation, models 20-471, 204015. The end walls are supported by the car frame through the inclined or vertical posts.

General view of such a car using the example of wagon for pellets transportation is shown in the (Fig. 1).

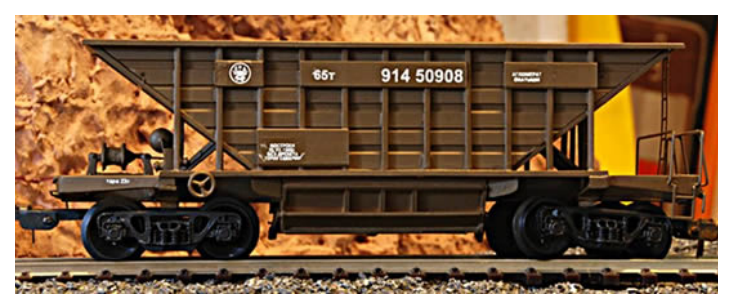

Fig. 1. The hopper car for transportation of pellets model 20-471

Owners of this type of rolling stock are interested in their maximal use, i.e. expansion of the range of cargoes and, accordingly, reduction of empty running and acceleration of car turnaround. A weighty argument for the enterprises using such cars is the possibility of their unloading on the car dumper.

A stationary rotary-type car dumper VRS-134M [3] used in the research unloads gondola cars and other types of open wagons by turning them over. The car dumper works in complex with electric trolley-pusher and shunting device. (Fig. 2).

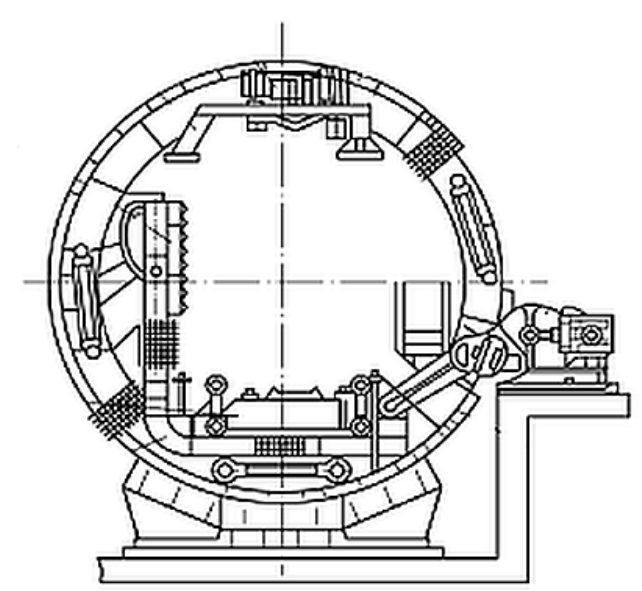

Fig. 2. The stationary rotary-type car dumper VRS-134M

The loaded cars are supplied by the car pusher and positioned on the platform inside the rotor of the car dumper. In the initial period of rotation of the rotor, the bridge with a car is shifted towards side wall of the car dumper until the car contacts it. With further rotation of the rotor, the cradle directed by the rollers in curved slots and crank rods, under the action of gravity moves with the car to the vibrator stops. In the overturned position, the car completely rests on the vibrators and the side wall of the car dumper. When the vibrators are turned on, the remaining of cargo is dumped out from the sides and bottom of the car.

The scheme of the car interaction with the parts of the car dumper is essential for subsequent analysis. The side wall of the car, without bending, rests on the side wall of the car dumper, and the upper top chord is fixed by three stops of the vibrators - the middle and the two outer ones. When the car is turned over, they serve as supports for the car body. 
Design feature of the open-type hopper cars is their short base and, correspondingly, a shorter body. At the present time there is no method of estimating the influence of external factors on the elements of such cars when unloading on car dumper. In order to do this, it is necessary to know all the actual operational loads, however, there are no regulatory recommendations for their evaluation in expanded form. Thus, it is necessary to develop the corresponding methodology for both placing hopper cars in the car dumper, as well as for determining the actual operational loads.

\section{Problem definition}

The article is devoted to the research of the adaptation of short-base hopper cars to the conditions of unloading the bulk cargoes using the method of overturning and the development of methodology for evaluating the design integrity of the open-type hopper cars when unloading them on the car dumper. This will solve practical problems: reducing downtime, turnover of hopper cars and, ultimately, reducing the operating costs and increasing the economic efficiency of their use.

\section{Analysis of research and publications}

General requirements to the types of loads acting on the car when unloading on the car dumper are given in the «Norms ...» [4] and DSTU GOST 33211:2017 [5]. According to this, the side walls of gondola cars and open hoppers are calculated for the simultaneous action of vertical and horizontal forces evenly distributed over the chord width at a length of $0.8 \mathrm{~m}$ in the middle part of the chord between the neighboring posts.

The calculated vertical force per span $q_{\mathrm{v}}, \mathrm{MN}$, is calculated using the following formula:

$$
q_{\mathrm{v}}=\frac{Q_{\mathrm{gr}}}{n}
$$

where $Q_{\mathrm{gr}}-$ is the gross gravity force of car, $\mathrm{MN}$; $n \quad-\quad$ is the number of stops of car dumper.

The horizontal transverse force is taken to equal to $0.25 q_{\mathrm{v}}$

The requirements for cars during strength tests in the process of loading and unloading operations are given in the GOST 33788-2016 [6], and the general requirements for ensuring the safety of freight cars are regulated by the DSTU GOST 22235-2015 [7].

Scientists in Russia [8-9] and in Ukraine [10] have dealt with the issues related to the improvement of methods for evaluating the strength of gondola car bodies.

Thus, in the work [8], calculation schemes for distribution of inertial loading from bulk cargo have been developed and a formula for determining the distribution of dynamic pressure along the height has been obtained. The calculation schemes were obtained by analyzing the interaction of the car dumper and gondola car with obtaining oscillograms of stresses in the structural elements.

The work [9] studies unloading process with determining dynamic forces and accelerations acting on the body elements when unloading bulk cargo using the car dumper. The mathematical model of movement of the cardumper-gondola car body-bulk cargo system during unloading is specified, the influence of the system's rotation angle on the change in the loading value is investigated.

In the work [10] a new method of gondola cars unloading was developed and patented. It is based on the use of suspended car dumper, which practically eliminates all the drawbacks of the known machines and mechanisms designed to unload gondola cars in terms of power action on their bodies. Calculations of the strength of structural elements of the gondola car body when interacting with this car dumper are presented.

The proposed methodologies for calculating gondola car under the action of inertial loading of bulk cargo and from the action of forces during unloading on the car dumper make it possible to increase the reliability of calculating the strength of gondola car design.

\section{Purpose and objectives of research}

All previously discussed methodologies [8-10] consider the scheme of interaction of a gondola car with the parts of car dumper. Therefore, the task of development of the model and engineering methodology for calculating the bodies of short-based hopper cars is the relevant one. In the course of the work complex, it is supposed to evaluate design strength of hopper cars and correspondence of the strength characteristics of the elements of regulatory documentation based on theoretical and experimental studies.

The possibility of unloading on the car dumper of the cars transporting the following cargoes is considered: crushed stone (granite and limestone), limestone, clay, granulated slag, coal, sand. The cargo must freely spill out of the body, not freeze or stick to it. In the last case the excessive load is applied to the upper part of the body when the car is overturned.

Car bodies are made of various materials (steel grades), the least durable among them is the steel grade 09G2 of strength class 295. The yield strength for it is $\sigma \mathrm{t}=305 \mathrm{MPa}[4$, table 11], the permissible stress of the I design mode is $[\sigma]=0 ; 90 \sigma \mathrm{t}=274 \mathrm{MPa}$ for the ridge and pivot beams and $[\sigma]=0 ; 95 \sigma \mathrm{t}=290 \mathrm{MPa}$ for other elements of the body [4, table 10.1].

\section{Main part of the research}

Solution of the assigned tasks was carried out by combining analytical methods, numerical modeling and field experiments.

The calculation is carried out using finite element analysis. Geometric models of the body were automatically divided into flat finite elements of the «cover» type (resisting both deformations in the proper plane, as well as bending deformations) with a characteristic edge size $50 \mathrm{~mm}$. The plating of side walls 
is excluded from geometric models, since, not being rigidly connected with the wall frame, it does not receive the loads acting on the car body.

The geometrical model and the loading scheme of the body elements are shown on the example of the car for transportation of pellets of the model 20-471 (Fig. 3).

It is assumed that the body rests on the middle and one of the outer stops. Loading groups: A, B - are hinged bearings of the pivoted joints, $\mathrm{C}$ - are dummy movable sliding bearings of side wall, $\mathrm{D}$ - is the gravity force of design, E - is the pressure on outer stop, F- is the pressure on the middle stop.

The stress state is estimated according to the values of the equivalent stresses Mises $\sigma_{\text {eq }}$ acting on the external surfaces of the elements:

$$
\sigma_{\text {экв }}=\frac{1}{\sqrt{2}} \sqrt{\begin{array}{l}
\left(\sigma_{x}-\sigma_{y}\right)^{2}+\left(\sigma_{y}-\sigma_{z}\right)^{2}+ \\
+\left(\sigma_{z}-\sigma_{x}\right)^{2}+6\left(\tau_{x y}^{2}+\tau_{y z}^{2}+\tau_{z x}^{2}\right)
\end{array}}
$$

where $\sigma_{x}, \sigma_{y}, \sigma_{z}, \tau_{x y}, \tau_{y z}, \tau_{z x}-$ are the components of stress tensor.

The pattern of distributions of stress arising in the elements of car bodies when unloading on the car dumper is shown in the (Fig. 5-7).

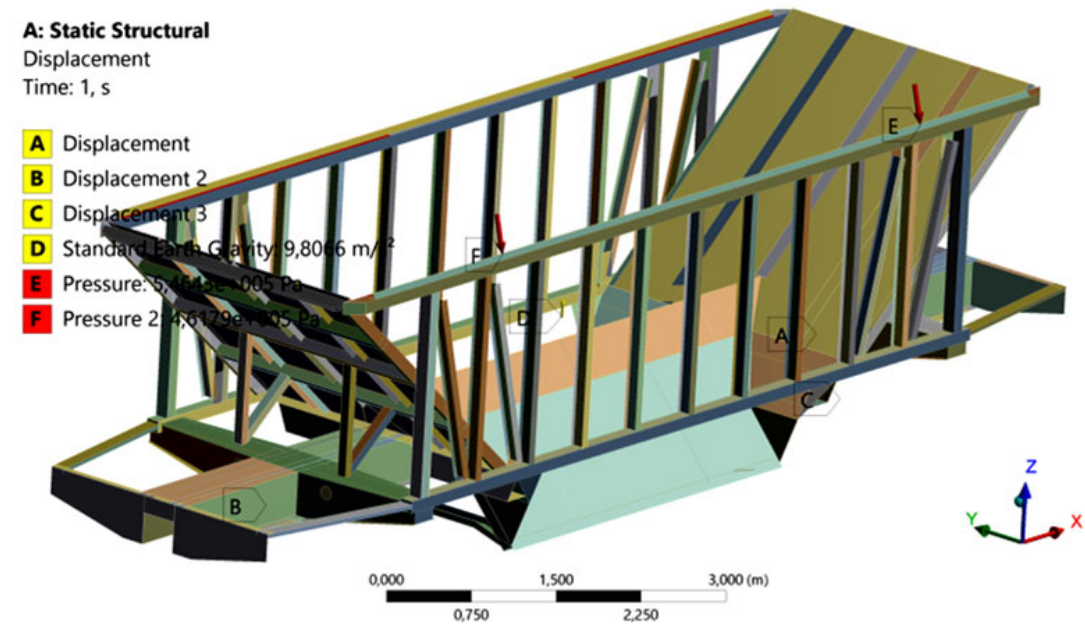

Fig. 3. Loads and supports for the car body $20-471$ at unloading on the car dumper

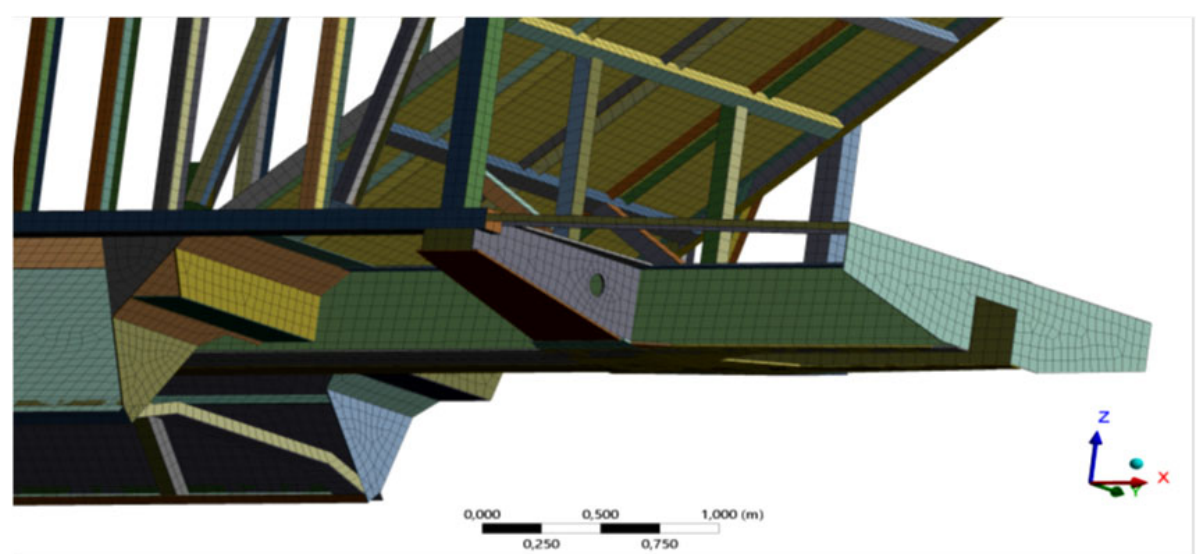

Fig. 4. The fragment of the finite element model of the console part of the car body 20-471. The console part of the body

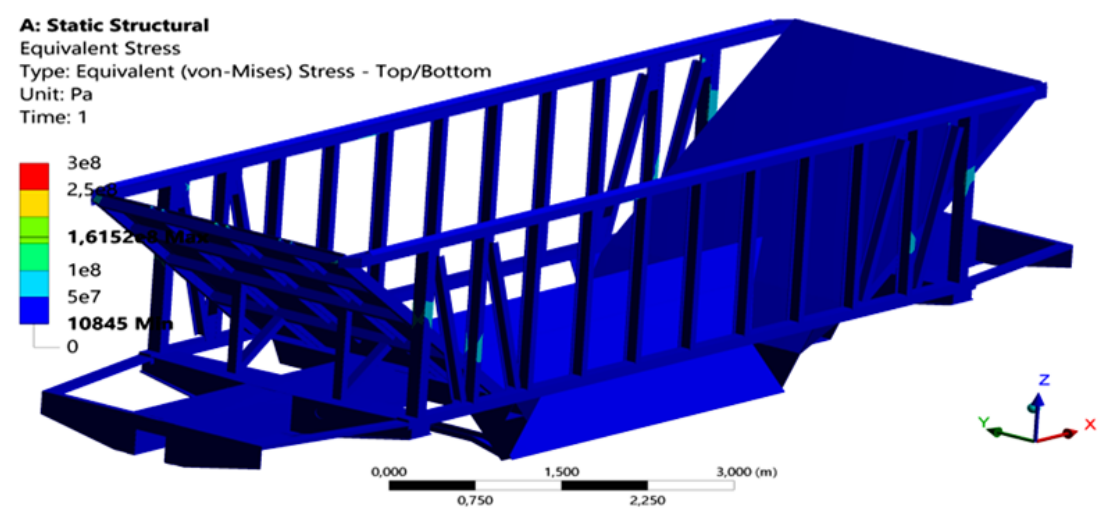

Fig. 5. Stresses in the car body 20-471 when unloading on the car dumper 


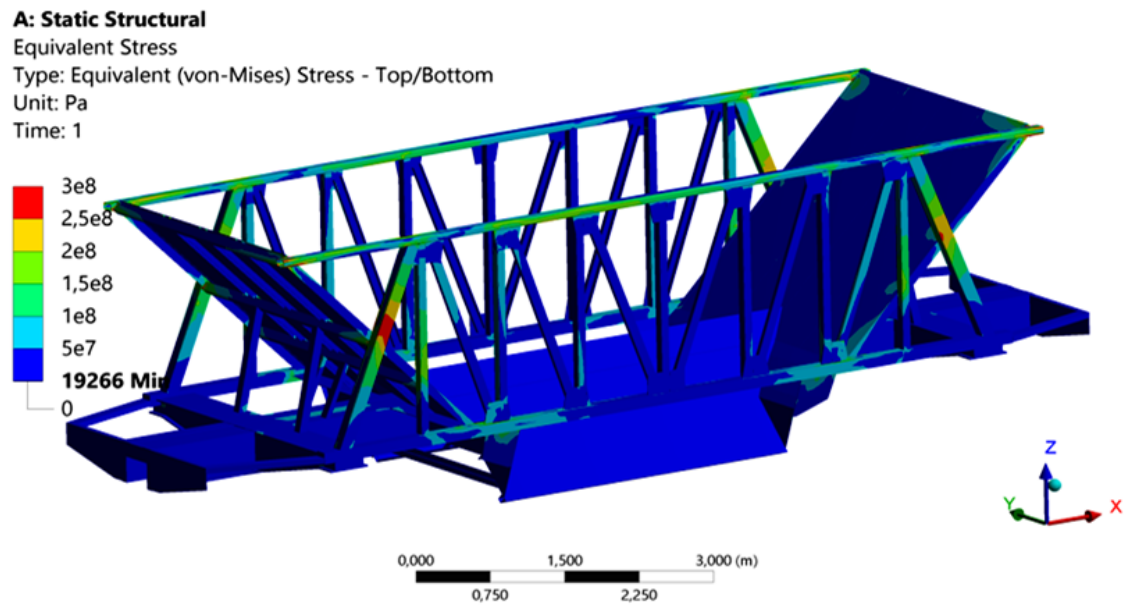

Fig. 6. Stresses in the car body 20-4015 when unloading on the car dumper

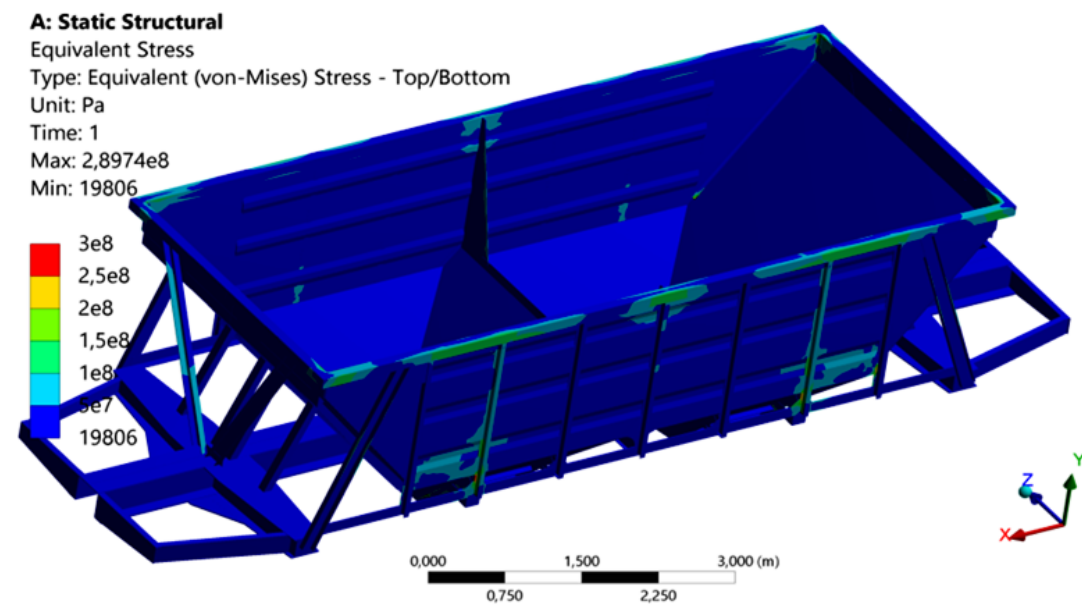

Fig. 7. Stresses in the car body 19-758-01 when unloading on the car dumper

Strength of the car body for cement transportation of the model 19-758-01 in the general case meets the requirements [4]. The stresses do not exceed $160 \mathrm{MPa}$ (when the admissible ones $[\sigma]=290 \mathrm{MPa}$ ), except for the concentration area on the side wall post, where they are equal to $224 \mathrm{MPa}$.

Strength of the car body for pellets transportation 20-471 meets the requirements [4]. The stresses reach the values of $250 \ldots 272 \mathrm{MPa}$ (the top and bottom chords, posts and trusses of the side walls, the bin jumper), which is less than the permissible $[\sigma]=290 \mathrm{MPa}$. The dimensions of the areas of construction with stresses greater than $250 \mathrm{MPa}$ are relatively small, less than $100 \mathrm{~mm}$.

The stresses in the side wall truss and at the base of the cantilever portion of the top chord in the car for transportation of pellets of model 20-4015, reaches the yield point. The strength of the other body elements meets the requirements [4]. This car can be unloaded on the car dumper, if it is excluded the possibility of nondumping out of cargo from the body (for example, unloading the cars on the car dumper in the warm season, avoiding adfreezing).
According to the results of calculations for the body strength of all the above-mentioned cars, it is allowed to unload them on the car dumper VRS-134M (provided that the body is supported by the middle and one of the outer stops along the entire length of the latter).

For the purpose of approbation of the proposed methodology of theoretical studies, experimental research with unloading of hopper cars on the car dumper was carried out. When unloading the experimental cars were loaded with limestone for technological needs of up to 66 tons and equipped with sensors for recording the stresses. After that, the loaded cars one by one were supplied to the car dumper VRS-134M and positioned under the outer and middle stops. Each car was turned over twice in a row (the second time - in the empty state), after which the cars were inspected to identify possible damages and loss of pivots. During the unloading process, the continuous stress recording was performed. An example of the stress recording in the body elements of the car for transportation of pellets, model 20-471 when unloading is shown in the (Fig. 6). Here the oscillogram is gridded: stresses in $\mathrm{MPa}-$ the time of turning the car in sec. 


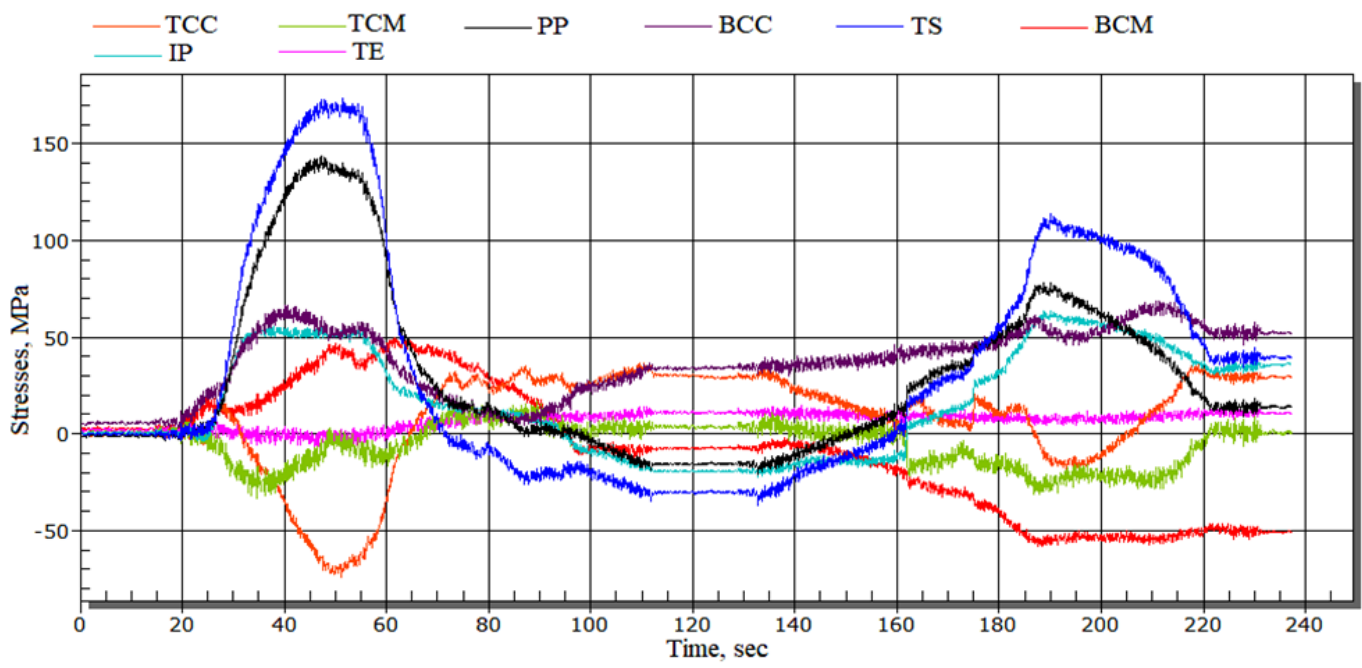

Fig. 8. The oscillogram of the stresses in the car body $20-471$ when unloading on the car dumper

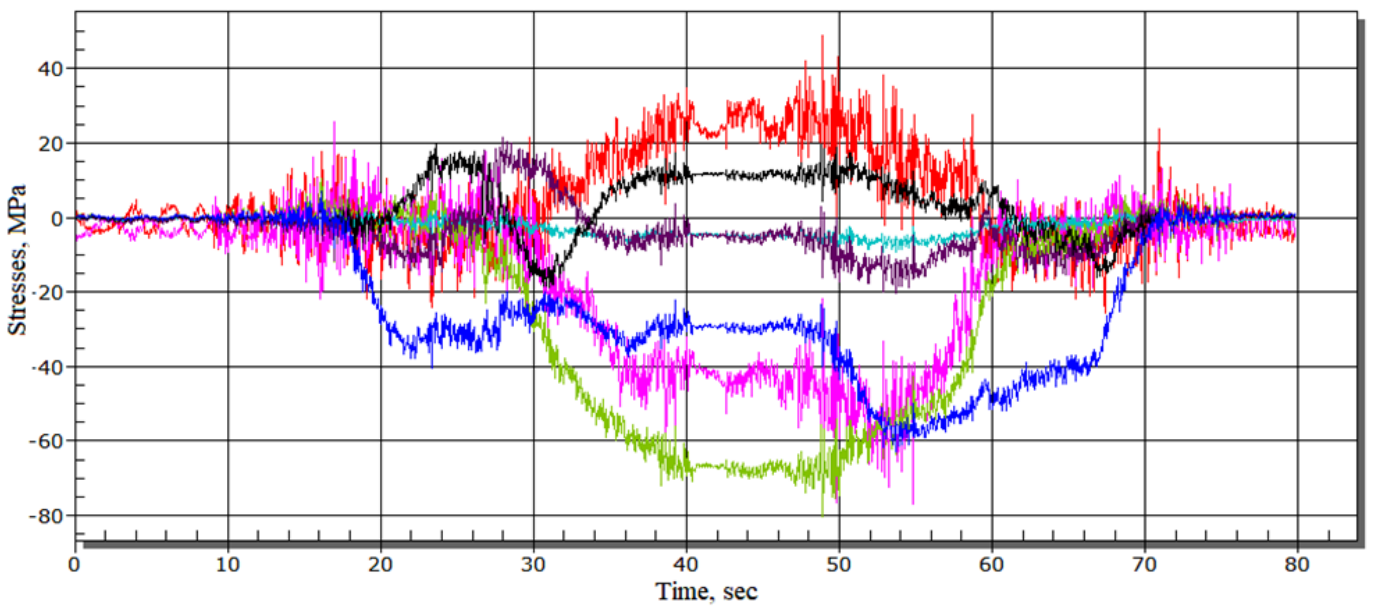

Fig. 9. Stresses in the car body 20-471, JSC DVM during overturning in an empty state

In the (Fig. 8,9) the following acronyms are accepted: TCC - is the top chord in the cantilever portion of the car, TCM - is the top chord in the middle part of the car, PP - is the pivot post, BCC - is the bottom chord in the cantilever portion of the car, TS - is the truss of the car side wall, BCM - is the bottom chord in the middle part of the car, IP - is the intermediate post, TE - is the truss of end wall.

On the oscillogram: $20-60$ seconds - is the turn of the loaded car and return to its initial position when unloading, 160-220 seconds - is the turn of the empty car and return to its initial position.

After processing the oscillograms according to the experiments and recalculating the results for full load capacity, the body strength of the all cars was evaluated.

When unloading on the car dumper, the maximum total stresses recalculated for the full load capacity of the car were as follows:

- for the model 19-758-01: 174.5 MPa (bin edge), 138.5 $\mathrm{MPa}$ (side wall post), $-118.8 \mathrm{MPa}$ (side wall truss);

- for the model 19-758-01: 174.5 MPa (bin edge), 138.5 $\mathrm{MPa}$ (side wall post), $-118.8 \mathrm{MPa}$ (side wall truss);
- for the model 20-4015: 211.4 MPa (bottom chord), -205.6 MPa (outer truss of the side wall post), 117.1 $\mathrm{MPa}$ (side wall post);

- $\quad$ for the model 20-471: 225.1 MPa (bottom chord), 176.0 $\mathrm{MPa}$ (side wall post).

It can be seen that for all elements of cars, the values of total stresses obtained are much lower than those allowed.

Thus, the stress values obtained in the course of experimental studies according to the developed methodology confirmed the results of earlier analytical calculations. And on the whole, the results of the research have shown the possibility of unloading the short base hopper cars on this type of car dumper.

\section{Conclusions}

As a result of the studies, it was developed the model and engineering methodology for calculating the bodies of short-based hopper cars for strength when unloading on the car dumper VRS-134M. In the process of performing the work, a scheme for interaction of the short-based hopper car with the parts of the car dumper is found out. It makes it possible to represent the 
computational model more accurately. Reliability of the obtained results is confirmed by a comparison of the results of the calculated and experimental data. Justification of the developed recommendations is confirmed by the positive test results.

The stresses in car bodies did not exceed the permissible $280 \mathrm{MPa}$ (steel 09G2S), i.e., the strength of the cars of the models $19-758-01,20-4015,20-471$ is sufficient for unloading on the car dumper VRS-134M provided that the cargo is free to dump out and the car is positioned under the outer and middle stops of the car dumper.

The proposed method can be used to calculate the bodies of the short-based hopper cars for strength when unloading on other types of the car dumper.

The research on the hopper cars adaptation to the conditions of unloading bulk cargoes using the method of overturning and strength estimation of their bodies made it possible to reliably estimate a stress condition of the car bodies and to justify the possibility of unloading these cars on the car dumper. The latter is essential for reducing the downtime and turnaround of hopper cars.

\section{References}

1. Freight railroad cars. Reference catalogue. Part 5. Hoppers.

2. Freight cars of gauge of $1520 \mathrm{~mm}$ of USSR railways (reference catalogue), Moscow, Transport, 177 p (1989)

3. N.P. Zhuravlev, O.B. Malikov Transport-Cargo Systems, Moscow, Marshrut, 367 p (2006)
4. Norms for the calculation and design of railroad cars of the Ministry of Railways of the gauge of $1520 \mathrm{~mm}$ (non-self-propelled), Moscow, GosNIIVVNIIZhT, 319 p (1996)

5. DSTU GOST 33211:2017. Freight cars. Requirements for structural strength and dynamic qualities. (GOST 33211-2014, IDT), Kyiv, SE UkrNDNC, $58 \mathrm{p}$ (2017)

6. GOST 33788-2016. Freight and passenger railcars. Methods of testing structural strength and dynamic performance, Moscow, Standardinform, 41 p (2016)

7. DSTU GOST 22235-2015. Freight cars fot $1520 \mathrm{~mm}$ gauge main railways. General requirements for safety in loading-unloading and shunting operations (GOST 22235-2010, IDT), Kyiv, SE UkrNDNC, 22 p (2015)

8. A.V. Dodonov, I.A. Hilov, A.A. Bityuk Improvement of the method of calculating the strength of the gondola body structure of the gondola during unloading on the wagon breaker. Approval of new methods of freight wagons designing: a collection of scientific works. Engineering center of car building, St. Petersburg, 11 (2012)

9. G.I. Petrov, I.V. Chepurnenko, O.I. Panachev Adaptation of the mathematical model of the gondola to the conditions of body unloading by overturning, Transport business of Russia, Moscow, 1 (2017)

10. R.I. Visnyak Determination of the strength of the body of the gondola during unloading of bulk cargoes, Author's abstract of candidate's thesis (Engineering), Kharkiv, UkrSURT, 20 p (2003) 